Research Article

\title{
Synchronization Analysis of a Class of Neural Networks with Multiple Time Delays
}

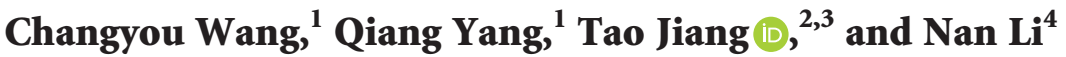 \\ ${ }^{1}$ College of Applied Mathematics, Chengdu University of Information Technology, Chengdu 610225, China \\ ${ }^{2}$ Control Engineering College, Chengdu University of Information Technology, Chengdu 610225, China \\ ${ }^{3}$ State Key Laboratory of Robotics, Shenyang Institute of Automation, Chinese Academy of Sciences, Shenyang, \\ Liaoning 110169, China \\ ${ }^{4}$ Department of Applied Mathematics, Southwestern University of Finance and Economics, Chengdu 610074, China
}

Correspondence should be addressed to Tao Jiang; jiangtop@126.com

Received 6 February 2021; Revised 7 March 2021; Accepted 27 March 2021; Published 8 April 2021

Academic Editor: Niansheng Tang

Copyright (c) 2021 Changyou Wang et al. This is an open access article distributed under the Creative Commons Attribution License, which permits unrestricted use, distribution, and reproduction in any medium, provided the original work is properly cited.

In this paper, we study the synchronization of a new fractional-order neural network with multiple delays. Based on the control theory of linear systems with multiple delays, we get the controller to analyse the synchronization of the system. In addition, a suitable Lyapunov function is constructed by using the theory of delay differential inequality, and some criteria ensuring the synchronization of delay fractional neural networks with Caputo derivatives are obtained. Finally, the accuracy of the method is verified by a numerical example.

\section{Introduction}

Since Leibniz and L'Hospital first proposed fractional calculus in 1675 , people have done a lot of pioneering work in the field [1]. These research studies involve many fields of science and engineering, such as electromagnetic wave, bioengineering, viscoelastic system, heat conduction, dielectric polarization, and robot [2-6]. Compared with the classical integer-order neural network, the fractional-order system can describe the relationship between input and output signals of neural networks and has better memory and heredity [7-11]. Therefore, it is very interesting and important to study the application and theory of fractionalorder systems.

Moreover, there are many research studies on the dynamic behavior in complex networks, especially synchronization problems, which is an important dynamic phenomenon in complex neural networks. The synchronization of neural networks means that the state of neural network nodes tends to be consistent with time. Over the past two decades, many scholars have developed various technologies in this research field. More and more synchronization schemes have been proposed, such as projection synchronization, lag synchronization, complete synchronization, antisynchronization, and robust synchronization (see, e.g., [12-18] and the references therein). For instance, in [12], the authors discussed a fractional-order 3dimensional neural network and obtained some conditions to ensure the projection synchronization of the new system by using the computer simulations and fractional calculus theory. The authors in [14] investigated a class of $n$ dimensional chaotic systems with uncertain parameters and discussed the complete synchronization of chaotic attractors for these systems. In 2014, Yu et al. [16] investigated the global projective synchronization of a fractional-order neural network and obtained some new criteria to realize projective synchronization of the fractional-order neural networks by combining open loop control and adaptive 
control. In 2017, Wang et al. [18] studied a fractional-order coupled neural network and achieved several criteria of robust synchronization for the proposed network.

On the contrary, time delay is inevitable in many complex systems, which has a significant impact on the dynamic characteristics of the system, such as oscillation, stability, and synchronization. Therefore, the study of neural network model with time delay has more important theoretical and practical significance. Recently, more and more scholars have paid attention to the research of neural networks with fractional derivative [19-27]. In [19], the Riemann-Liouville fractional-order neural networks with timevarying delays are studied. By using the Lyapunov functional method, some stability criteria of the proposed neural networks with fractional derivative and delays are achieved. In 2010, Li et al. [21] investigated a chaotic delayed neural network with stochastic perturbations and achieved some criteria to ensure the exponential synchronization for the addressed neural networks in terms of Lyapunov-Krasovskii functional method. Zheng et al. [22] studied the projective synchronization of a memristor-based delayed fractionalorder neural network by using differential inclusion theory and set-valued map. Udhayakumar et al. [23] considered a fractional-order delayed complex-valued neural networks and afforded some conditions ensuring the projective synchronization of the addressed systems by applying Lyapunov-Krasovskii functional approach and linear matrix inequalities. Wang et al. [24] studied a class of multidirectional memory neural networks with time delay and achieved some criteria of the synchronization within a fixed time for master-slave systems via utilizing the Lyapunov stability theory. More recently, in 2019, Hu et al. [25] discussed a class of time-invariant uncertainty delayed fractional-order neural networks and obtained several global synchronization criteria of the delayed neural networks model by using fractional-order integral Jensen's inequality and Lyapunov-Krasovskii functions. In 2020, You et al. [27] investigated the discrete-time fractional-order delayed complex-valued neural networks and achieved some criteria to ensure the global Mittag-Leffler synchronization for the proposed delayed master-slave systems by devising an effective control scheme and applying Lyapunov's direct method. In 2021, Song et al. [28] considered the problem on passive filter design for fractional-order quaternion-valued neural networks with neutral delays and external disturbance and derived some sufficient conditions to confirm the augmented filtering dynamic system to be stable and passive with an expected dissipation by constructing Lyapunov-Krasovskii functional and using the inequality technique. For more research on neural networks with time delay, refer to [29-35].

Furthermore, it is not difficult to see that most of the above literature based on the synchronization of fractionalorder neural networks do not consider the influence of multiple time delays. It should be pointed out that the fractional-order neural network model with multiple time delays can better describe the objective laws in the nervous system. However, there are only few results on the synchronization of fractional-order neural networks with multiple time delays in existing literature [36-39] because it is very difficult to study the stability of nonlinear fractionalorder neural networks with multiple delays. Motivated by the above considerations, our aim in this paper is to investigate the global Mittag-Leffler synchronization for a class of fractional-order neural networks with multiple time delays. Based on the Lyapunov stability theory and comparison principle of the fractional-order differential equation with delays, some criteria ensuring the synchronization of the fractional-order neural networks with multiple time delays are achieved. This paper is organized as follows. In Section 2, some definitions and new fractional-order differential inequalities are introduced, and the proposed new systems are given. In Section 3, some criteria ensuring the globally asymptotic synchronization for the addressed systems are obtained. The effectiveness and feasibility of the theoretical results are shown by an example in Section 4 . The obtained results and future research topics are provided in Section 5 .

\section{Preliminaries and System Description}

In this section, we first give some lemmas and definitions of fractional-order calculus, which will be used in the proof of main results in this paper. In addition, a fractional-order neural networks system with multiple time delays is described.

Definition 1. (see [40]). The Gamma function is defined as

$$
\Gamma(\alpha)=\int_{0}^{\infty} e^{-t} t^{\alpha-1} \mathrm{~d} t
$$

It is easy to see that the Gamma function satisfies the recursive relation $\Gamma(\alpha+1)=\alpha \Gamma(\alpha)$.

Definition 2. (see [40]). The Caputo fractional derivative of function $x(t)$ is defined as

$$
D_{0, t}^{\alpha} x(t)=D_{0, t}^{-(n-\alpha)} \frac{\mathrm{d}^{n}}{\mathrm{~d} t^{n}} x(t)=\frac{1}{\Gamma(n-\alpha)} \int_{0}^{t}(t-s)^{n-\alpha-1} x^{n}(s) \mathrm{d} s,
$$

where $\alpha$ represents the order of the derivative and $n-1<\alpha \leq n$. The fractional-order derivative $D^{\alpha}$ discussed in this paper refers to the Caputo derivative $D_{0, t}^{\alpha}$.

Definition 3. (see [41]). Set $\alpha>0, z \in C$, then

$$
E_{\alpha}(z)=\sum_{k=0}^{\infty} \frac{z^{k}}{\Gamma(k \alpha+1)}
$$

is called a single parameter Mittag-Leffler function.

Definition 4. (see [41]). Set $\alpha>0, \beta>0$, and $z \in C$, then

$$
E_{\alpha, \beta}(z)=\sum_{k=0}^{\infty} \frac{z^{k}}{\Gamma(k \alpha+\beta)}
$$


is called a two-parameter Mittag-Leffler function. According to Definition 3, it is not difficult to see $E_{\alpha}(z)=E_{\alpha, 1}(z)$ and $E_{1,1}(z)=e^{z}$.

Lemma 1. (see [40]). If $V(t) \in C^{1}([0,+\infty), R), 0<\alpha<1$, and satisfies $D^{\alpha} V(t) \leq-\lambda V(t)$, then

$$
V(t) \leq V(0) E_{\alpha}\left(-\lambda t^{\alpha}\right), \quad t \geq 0,
$$

where $\lambda$ is a positive number.

Lemma 2 (see [41]). Let $\alpha<2, \beta$ be an any real number, $(\pi \alpha / 2)<\mu<\min \{\pi, \pi \alpha\}$, then there exists a positive constant C so that

$$
\left|E_{\alpha, \beta}(z)\right| \leq \frac{C}{1+|z|}, \quad(\mu \leq|\arg (z)| \leq \pi),|z|>0 .
$$

Next, we discuss a class of fractional-order neural networks model with multiple time delays which is described by the following differential equation:

$$
\begin{aligned}
D^{\alpha} x_{i}(t)= & -a_{i} x_{i}(t)+\sum_{j=1}^{n} b_{i j} f_{j}\left(x_{j}(t)\right) \\
& +\sum_{m=1}^{n} \sum_{j=1}^{n} c_{i j}^{(m)} f_{j}\left(x_{j}(t-m \tau)\right)+I_{i}, \quad i=1,2, \ldots, n,
\end{aligned}
$$

or in the following vector form:

$$
D^{\alpha} x(t)=-A x(t)+B f(x(t))+\sum_{m=1}^{n} C_{m} f(x(t-m \tau))+I,
$$

where $x(t)=\left(x_{1}(t), \ldots, x_{n}(t)\right)^{T} \in R^{n}$ represents the state variable of the neuron at time $t, \tau$ denotes the communication delay of the neuron, $A=\operatorname{diag}\left(a_{1}, a_{2}, \ldots, a_{n}\right)$, and $a_{i}$ is the self-inhibition rate of $i$-th neuron. $B=\left(b_{i j}\right)_{n \times n}$, and $b_{i j}$ denotes the connection weights. $C_{m}=\left(c_{i j}^{(m)}\right)_{n \times n}$, in which $m$ is a positive integer and $c_{i j}^{(m)}$ indicates the delayed connection weights. $f(x(t))=\left(f_{1}\left(x_{1}(t)\right), \ldots, f_{n}\left(x_{n}(t)\right)\right)^{T}$, and $f_{i}\left(x_{i}(t)\right)$ is the excitation function of the $i$-th neuron. $I=\left(I_{1}, \ldots, I_{n}\right)^{T}$, and $I_{i}$ is the network deviation of the $i$-th neuron.

\section{Synchronization Scheme}

In this part, we give some criteria for the globally asymptotic synchronization of the proposed neural networks with multiple delays. The model (7) is taken as the master system, and the following delayed fractional-order differential equation is taken as the slave system:

$$
\begin{aligned}
D^{\alpha} y_{i}(t)= & -a_{i} y_{i}(t)+\sum_{j=1}^{n} b_{i j} f_{j}\left(y_{j}(t)\right) \\
& +\sum_{m=1}^{n} \sum_{j=1}^{n} c_{i j}^{(m)} f_{j}\left(y_{j}(t-m \tau)\right)+u_{i}(t)+I_{i},
\end{aligned}
$$

$$
\begin{aligned}
D^{\alpha} y(t)= & -A y(t)+B f(y(t))+\sum_{m=1}^{n} C_{m} f(y(t-m \tau)) \\
& +u(t)+I
\end{aligned}
$$

where $y(t)=\left(y_{1}(t), \ldots, y_{n}(t)\right)^{T} \in R^{n}$ is the state variable of the slave system $(9)$ and $u(t)=\left(u_{1}(t), \ldots, u_{n}(t)\right)^{T}$ refers to the synchronous controller designed below. Other parameters have the same meaning as those given in the master system (7).

Let the error vector be $e_{i}(t)=y_{i}(t)-x_{i}(t)$, and from the master system (7) and the slave system (9), the expression of the error system can be obtained as

$$
\begin{aligned}
D^{\alpha} e_{i}(t)= & -a_{i} e_{i}(t)+\sum_{j=1}^{n} b_{i j}\left[f_{j}\left(y_{j}(t)\right)-f_{j}\left(x_{j}(t)\right)\right] \\
& +\sum_{m=1}^{n} \sum_{j=1}^{n} c_{i j}^{(m)}\left[f_{j}\left(y_{j}(t-m \tau)\right)-f_{j}\left(x_{j}(t-m \tau)\right)\right] \\
& +u_{i}(t),
\end{aligned}
$$

or in the following vector form:

$$
\begin{aligned}
D^{\alpha} e(t)= & -A e(t)+B\left[f(y(t))-f_{j}(x(t))\right] \\
& +\sum_{m=1}^{n} C_{m}[f(y(t-m \tau))-f(x(t-m \tau))]+u(t),
\end{aligned}
$$

where $e(t)=\left(e_{1}(t), \ldots, e_{n}(t)\right)^{T}$.

Obviously, the problem of asymptotic synchronization between the master system (7) and the slave system (9) is converted to the globally asymptotic stability problem of zero solution for the error system (11).

In order to achieve the criteria of the synchronization between the master system (7) and the slave system (9), a new state feedback controller with multiple delays is designed, as in the following form:

$$
u(t)=-K e(t)-\sum_{m=1}^{n} K_{m} e(t-m \tau)
$$

where $K=\left(k_{i j}\right)_{n \times n}$ and $K_{m}=\left(k_{i j}^{(m)}\right)_{n \times n}$ represent the control gain matrix. Therefore, from controller (13), the error system (11) can be written as

$$
\begin{aligned}
D^{\alpha} e_{i}(t)= & -a_{i} e_{i}(t)+\sum_{j=1}^{n} b_{i j}\left[f_{j}\left(y_{j}(t)\right)-f_{j}\left(x_{j}(t)\right)\right] \\
& +\sum_{m=1}^{n} \sum_{j=1}^{n} c_{i j}^{(m)}\left[f_{j}\left(y_{j}(t-m \tau)\right)-f_{j}\left(x_{j}(t-m \tau)\right)\right] \\
& +\left[-\sum_{j=1}^{n} k_{i j} e_{j}(t)-\sum_{m=1}^{n} \sum_{j=1}^{n} k_{i j}^{(m)} e_{j}(t-m \tau)\right] .
\end{aligned}
$$

Next, we give the main conclusion and its proof. 
Theorem 1. If for any $u(t), v(t) \in C([0,+\infty), R)$ exists in $L_{j}>0, j=1, \ldots, n$, such that $\left|f_{j}(u)-f_{j}(v)\right| \leq L_{j}|u-v|$, then when $a_{i}>0, i=1, \ldots, n$, the master-slave systems (7) and (9) are globally asymptotic synchronization under the controller (13), where the control gain matrix is shown as follows:

$$
K=|B| \operatorname{diag}\left(L_{1}, \ldots, L_{n}\right), K_{m}=\left|C_{m}\right| \operatorname{diag}\left(L_{1}, \ldots, L_{n}\right) .
$$

Proof. When $e_{i}(t)<0$, we have

$$
\begin{aligned}
D^{\alpha}\left|e_{i}(t)\right| & =\frac{1}{\Gamma(1-\alpha)} \int_{0}^{t} \frac{\left|e_{i}(s)\right|^{\prime}}{(t-s)^{\alpha}} \mathrm{d} s \\
& =-\frac{1}{\Gamma(1-\alpha)} \int_{0}^{t} \frac{e_{i}^{\prime}(s)}{(t-s)^{\alpha}} \mathrm{d} s=-D^{\alpha} e_{i}(t) .
\end{aligned}
$$

When $e_{i}(t)=0$, we have $D^{\alpha}\left|e_{i}(t)\right|=0$. Also, when $e_{i}(t)>0$, it holds that

$$
\begin{aligned}
D^{\alpha}\left|e_{i}(t)\right| & =\frac{1}{\Gamma(1-\alpha)} \int_{0}^{t} \frac{\left|e_{i}(s)\right|}{(t-s)^{\alpha}} \mathrm{d} s \\
& =\frac{1}{\Gamma(1-\alpha)} \int_{0}^{t} \frac{e_{i}^{\prime}(s)}{(t-s)^{\alpha}} \mathrm{d} s=D^{\alpha} e_{i}(t) .
\end{aligned}
$$

To sum up, it follows that

$$
D^{\alpha}\left|e_{i}(t)\right|=\operatorname{sgn}\left(e_{i}(t)\right) D^{\alpha} e_{i}(t) .
$$

Construct the following auxiliary function:

$$
V(t)=\sum_{i=1}^{n}\left|e_{i}(t)\right| .
$$

From equations (15) and (18), the Caputo fractionalorder derivative of $V(t)$ along the solutions of system (14) can be obtained as

$$
\begin{aligned}
D^{\alpha} V(t)= & \sum_{i=1}^{n} D^{\alpha}\left(\left|e_{i}(t)\right|\right)=\sum_{i=1}^{n} \operatorname{sgn}\left(e_{i}(t)\right)\left\{-a_{i} e_{i}(t)+\sum_{j=1}^{n} b_{i j}\left[f_{j}\left(y_{j}(t)\right)-f_{j}\left(x_{j}(t)\right)\right]+\sum_{m=1}^{n} \sum_{j=1}^{n} c_{i j}^{(m)}\left[f_{j}\left(y_{j}(t-m \tau)\right)-f_{j}\left(x_{j}(t-m \tau)\right)\right]\right. \\
& \left.+\left[-\sum_{j=1}^{n} k_{i j} e_{j}(t)-\sum_{m=1}^{n} \sum_{j=1}^{n} k_{i j}^{(m)} e_{j}(t-m \tau)\right]\right\} \\
= & \sum_{i=1}^{n} \operatorname{sgn}\left(e_{i}(t)\right)\left\{-a_{i} e_{i}(t)+\sum_{j=1}^{n} b_{i j}\left[f_{j}\left(y_{j}(t)\right)-f_{j}\left(x_{j}(t)\right)\right]+\sum_{m=1}^{n} \sum_{j=1}^{n} c_{i j}^{(m)}\left[f_{j}\left(y_{j}(t-m \tau)\right)-f_{j}\left(x_{j}(t-m \tau)\right)\right]\right. \\
& \left.+\left[-\sum_{j=1}^{n}\left|b_{i j}\right| L_{j} e_{j}(t)-\sum_{m=1}^{n} \sum_{j=1}^{n} C_{i j}^{(m)} L_{j} e_{j}(t-m \tau)\right]\right\}, \\
\leq & \sum_{i=1}^{n}\left\{-a_{i}\left|e_{i}(t)\right|+\sum_{j=1}^{n}\left|b_{i j}\right| L_{j}\left|e_{j}(t)\right|+\sum_{m=1}^{n} \sum_{j=1}^{n}\left|c_{i j}^{(m)}\right| L_{j}\left|e_{j}(t-m \tau)\right|+\left[-\sum_{j=1}^{n}\left|b_{i j}\right| L_{j}\left|e_{j}(t)\right|-\sum_{m=1}^{n} \sum_{j=1}^{n}\left|c_{i j}^{(m)}\right| L_{j}\left|e_{j}(t-m \tau)\right|\right]\right\} \\
= & \sum_{i=1}^{n}\left(-a_{i}\left|e_{i}(t)\right|\right) \leq \sum_{i=1}^{n}-\lambda\left|e_{i}(t)\right|=-\lambda V(t),
\end{aligned}
$$

where $\lambda=\min _{1 \leq i \leq n} a_{i}$. From Lemma 1, we have

$$
V(t) \leq V(0) E_{\alpha}\left(-\lambda t^{\alpha}\right)
$$

By means of the definition of norm, we have

$$
\begin{gathered}
V(t)=\sum_{i=1}^{n}\left|e_{i}(t)\right|=\|e(t)\|, \\
V(0)=\sum_{i=1}^{n}\left|e_{i}(0)\right|=\|e(0)\| .
\end{gathered}
$$

Thus, it follows that

$$
\|e(t)\| \leq\|e(0)\| E_{\alpha}\left(-\lambda t^{\alpha}\right) .
$$

Let $z=-\lambda t^{\alpha},|\arg (z)|=\pi$; from Lemma 2, we can see that there is a positive constant $C$ such that

$$
\left|E_{\alpha}\left(-\lambda t^{\alpha}\right)\right| \leq \frac{C}{1+\left|-\lambda t^{\alpha}\right|} .
$$

From the above formula, it is easy to see that $\|e(t)\|$ asymptotically approaches zero when $t$ approaches infinity; that is, the master system (7) and the slave system (9) are globally asymptotic synchronization.

Remark 1. Compared with the related works in [33], in this paper, some criteria ensuring the globally asymptotic synchronization of the fractional-order neural networks model with multiple time delays are simpler, which will be beneficial to the application of the obtained result in practical problems. In additional, we also give the change processes of error with the different fractional orders $e_{i}(t)$ with the different fractional orders $\alpha$ in Figure 1. 


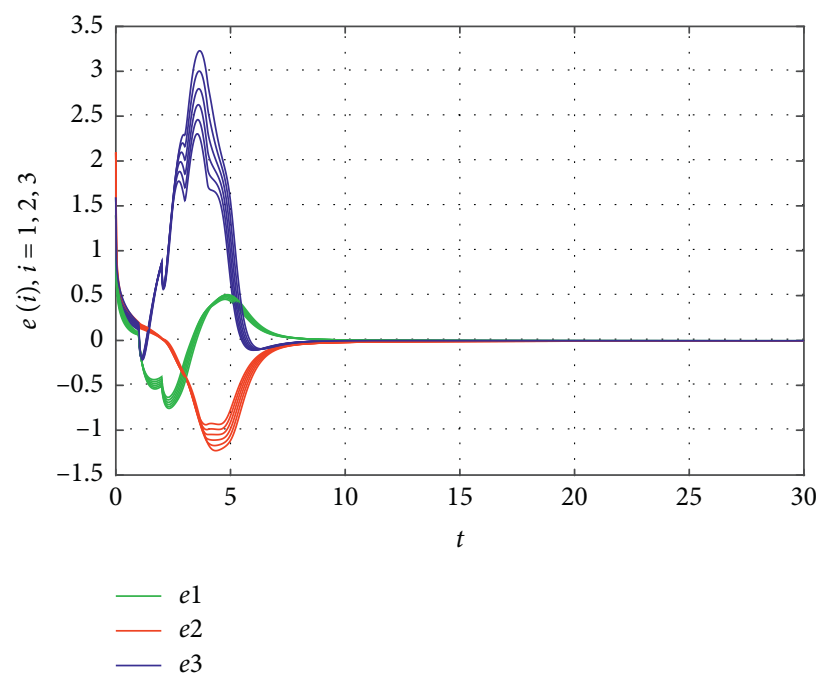

FIgURE 1: The change processes of error $e_{i}(t)$ under the different fractional orders $\alpha$.

\section{Numerical Example}

In this part, we provide an example to illustrate the effectiveness and feasibility of the theoretical results.

Example 1. Consider the following 3-dimensional fractional-order neural network with multiple delays (7):

$$
D^{\alpha} x(t)=-A x(t)+B f(x(t))+\sum_{m=1}^{2} C_{m} f(x(t-m \tau))+I .
$$

The parameters are selected as

$$
\begin{aligned}
A & =\operatorname{diag}\left(\begin{array}{lll}
1 & 1 & 1
\end{array}\right), \\
B & =\left[\begin{array}{ccc}
2 & -1.2 & 2 \\
2 & 1.71 & 1.13 \\
-2.75 & 2 & 1.1
\end{array}\right], \\
C_{1} & =\left[\begin{array}{ccc}
1 & -1.2 & 0 \\
1 & 1.71 & 1.13 \\
-2.75 & 0 & 1
\end{array}\right], \\
C_{2} & =\left[\begin{array}{ccc}
3 & -1.2 & 0 \\
3 & 1.71 & 1.15 \\
-3.75 & 0 & 1.1
\end{array}\right] .
\end{aligned}
$$

Let the parameters $\alpha=0.86, \tau=0.01, I=\left(I_{1}, I_{2}, I_{3}\right)^{T}=$ $(0,0,0)^{T}$ and the activation function vector $f(x(t))=\tanh$ $\left(x_{1}(t)\right), \tanh \left(x_{2}(t)\right), \tanh \left(x_{3}(t)\right)^{T}$. It can be obtained by simply computing that $L_{1}=L_{2}=L_{3}=1$, and the criteria of Theorem 1 are satisfied. Moreover, the controller return matrices $K, K_{1}$, and $K_{2}$ can be designed as

$$
\begin{gathered}
K=|B| L=\left[\begin{array}{ccc}
2 & 1.2 & 0 \\
2 & 1.71 & 1.13 \\
2.75 & 0 & 1.1
\end{array}\right], \\
K_{1}=\left|C_{1}\right| L=\left[\begin{array}{ccc}
1 & 1.2 & 0 \\
1 & 1.71 & 1.13 \\
2.75 & 0 & 1
\end{array}\right], \\
K_{2}=\left|C_{2}\right| L=\left[\begin{array}{ccc}
3 & 1.2 & 0 \\
3 & 1.71 & 1.15 \\
3.75 & 0 & 1.1
\end{array}\right] .
\end{gathered}
$$

The initial values of $x(t)$ and $y(t)$ are taken as

$$
\begin{aligned}
& x_{1}(t)=0.3, \\
& x_{2}(t)=0.1, \\
& x_{3}(t)=0.2, \quad t \in[-0.02,0], \\
& y_{1}(t)=1.7, \\
& y_{2}(t)=2.2, \\
& y_{3}(t)=1.8, \quad t \in[-0.02,0] .
\end{aligned}
$$

By employing the step-by-step iterative method for solving fractional differential difference equations and using MATLAB 7.0 software package, under the network parameters, controller gain, and the above initial values, we solve the numerical solutions of the master system (7), slave system (9), and error system (12), as shown in Figures 2 and 3. In Figure 2, we describe the state change processes of the master-slave systems (7) and (9) with the initial values (28) and (29). The state change processes of the error system (12) are shown in Figure 3. From Figure 3, it is easy to see that the master-slave systems (7) and (9) are globally Mittag-Leffler asymptotic synchronization, which verifies the rationality and effectiveness of the conditions given in Theorem 1 . 

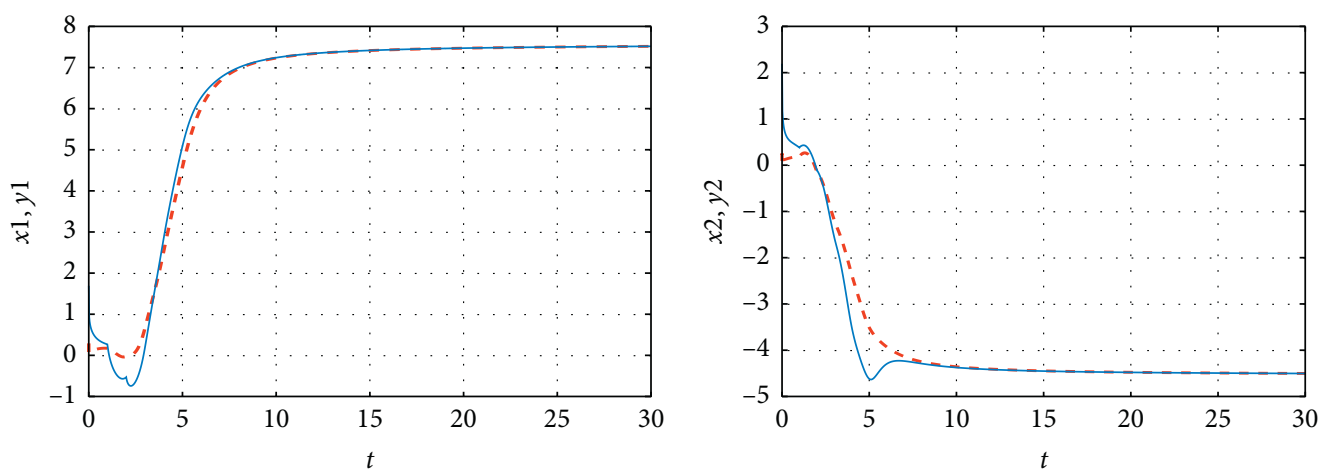

$---x 1$
$-y 1$

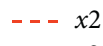

- $y 2$

(a)

(b)

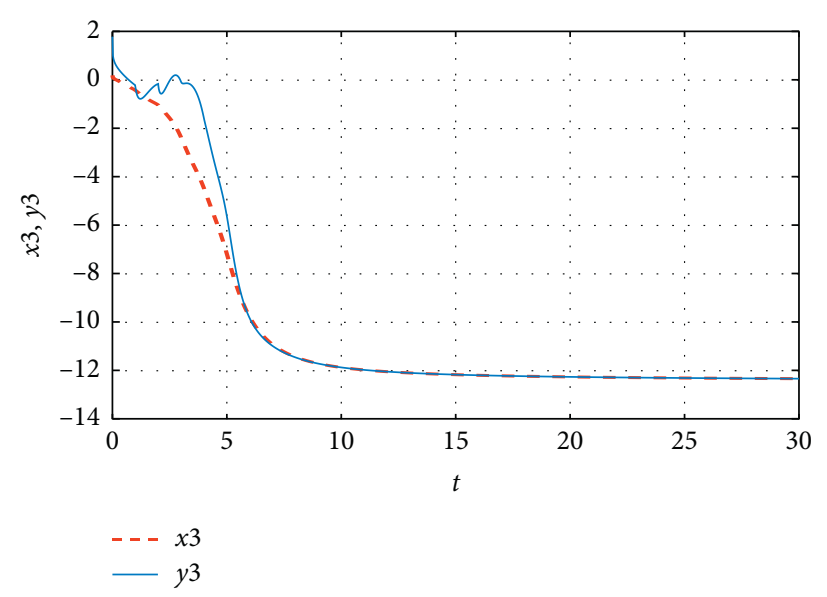

(c)

Figure 2: The change processes of $x(t)$ and $y(t)$ under the initial values (28) and (29).

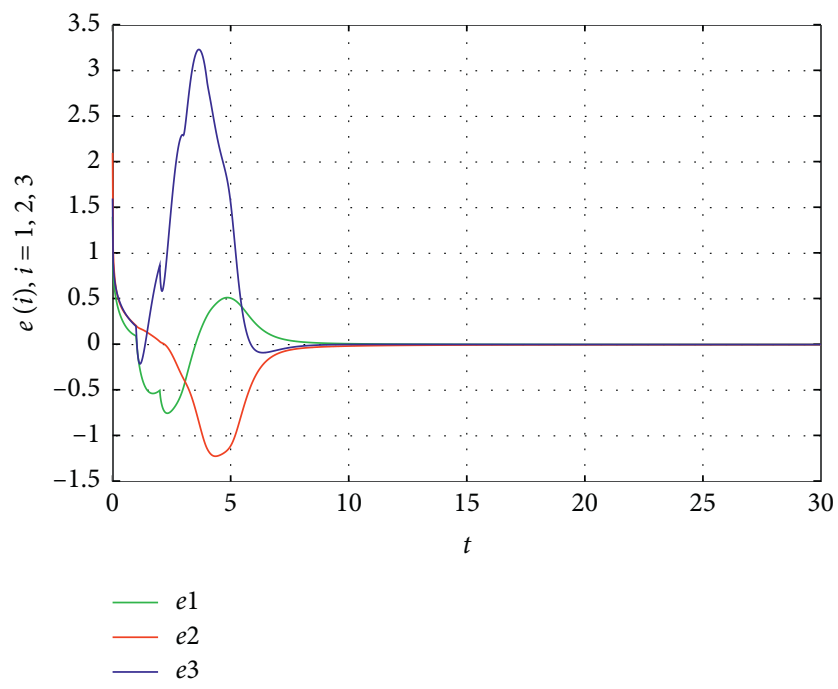

Figure 3: The change processes of error $e(t)$ under the initial values (28) and (29). 


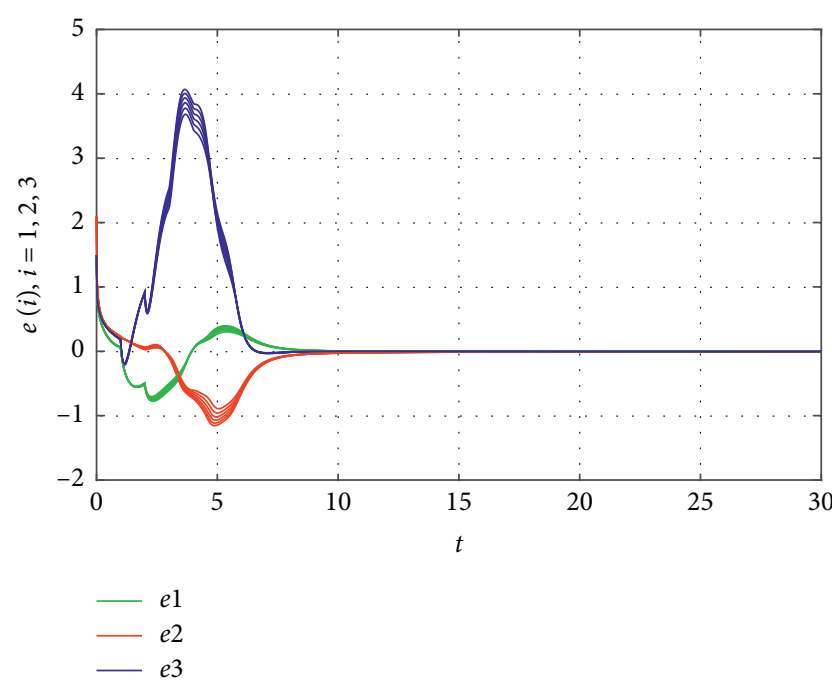

Figure 4: The change processes of error $e_{i}(t)$ under the different initial values.

To further verify that the master-slave systems (7) and (9) are globally Mittag-Leffler asymptotic synchronization, we choose different fractional orders and different initial values for simulation, and the results are shown in Figures 1 and 4 .

Obviously, from the above Figures 1 and 4, the order of fractional derivative and the initial value will not affect the global Mittag-Leffler synchronization of master-slave systems (7) and (9).

\section{Conclusion}

This paper discusses the global Mittag-Leffler synchronization of a new fractional-order neural network with multiple delays. In order to realize the global synchronization of the master-slave systems, we design a new multidelay state feedback controller. By constructing an appropriate Lyapunov function of the error system and based on the proposed control scheme with multiple time delays, sufficient conditions for global Mittag-Leffler synchronization of fractional-order neural networks with multiple time delays are given. In addition, the effectiveness of the proposed method is verified by numerical simulation.

It is very interesting to extend the results of this paper to the more general fractional-order neural network systems with multiple delays or time-varying delays by devising new suitable controllers. In the future, we intend to investigate the stability and synchronization of the more general neural network model with time-varying delays:

$$
\begin{aligned}
D^{\alpha} x_{i}(t)= & -a_{i} x_{i}(t)+\sum_{j=1}^{n} b_{i j} f_{j}\left(x_{j}(t)\right) \\
& +\sum_{m=1}^{n} \sum_{j=1}^{n} c_{i j}^{(m)} f_{j}\left(x_{j}\left(t-\tau_{m j}(t)\right)\right) \\
& +I_{i}, \quad i=1,2, \ldots, n,
\end{aligned}
$$

under appropriate assumptions.

\section{Data Availability}

The data used to support the findings of this study are available from the corresponding author on request.

\section{Conflicts of Interest}

The authors declare that they have no conflicts of interest.

\section{Authors' Contributions}

All authors contributed equally to each part of this work. All authors read and approved the final manuscript.

\section{Acknowledgments}

This work was supported by the Applied Basic Research Project of Sichuan Province (Grant no. 2018JY0480) of China, the Scientific Research Fund of Chengdu University of Information Technology (Grant no. KYTZ201820) of China, the State Key Laboratory of Robotics (Grant no. 2019O13) of China, and the Scientific Research Fund of Sichuan Provincial Science \& Technology Department (Grant no. 2021YFH0069) of China.

\section{References}

[1] I. Pondlubny, Fractional Differential Equations: An Introduction to Fractional Derivatives, Fractional Differential Equations, to Methods of Their Solution and Some of Their Applications, Academic Press, San Diego, CA, USA, 1998.

[2] C. Wang, R. Wang, S. Wang, and C. Yang, "Positive solution of singular boundary value problem for a nonlinear fractional differential equation," Boundary Value Problems, vol. 2011, Article ID 297026, 2011.

[3] C. Wang, H. Zhang, and S. Wang, "Positive solution of a nonlinear fractional differential equation involving Caputo derivative," Discrete Dynamics in Nature and Society, vol. 2012, Article ID 425408, 16 pages, 2012.

[4] S. Shi, M. Xiao, L. Rong et al., "Stability and bifurcation control of a neuron system under a novel fractional-order PD controller," Science China Technological Sciences, vol. 62, no. 12, pp. 2120-2129, 2019.

[5] S. Qureshi and S. Aziz, "Fractional modeling for a chemical kinetic reaction in a batch reactor via nonlocal operator with power law kernel," Physica A: Statistical Mechanics and Its Applications, vol. 542, Article ID 123494, 2020.

[6] H. You, Y. Shen, H. Xing, S. Yang, and S. Yang, "Optimal control and parameters design for the fractional-order vehicle suspension system," Journal of Low Frequency Noise, Vibration and Active Control, vol. 37, no. 3, pp. 456-467, 2018.

[7] A. Khan, K. Nasreen, and L. S. Jahanzaib, "Synchronization on the adaptive sliding mode controller for fractional order complex chaotic systems with uncertainty and disturbances," International Journal of Dynamics and Control, vol. 7, no. 4, pp. 1419-1433, 2019.

[8] X. Wu and L. Huang, "Pinning adaptive and exponential synchronization of fractional-order uncertain complex neural networks with time-varying delays," Neural Processing Letters, vol. 50, no. 3, pp. 2373-2388, 2019.

[9] P. Gong, "Distributed consensus of non-linear fractionalorder multi-agent systems with directed topologies," IET 
Control Theory \& Applications, vol. 10, no. 18, pp. 2515-2525, 2016.

[10] A. Wu and Z. Zeng, "Global mittag-leffler stabilization of fractional-order memristive neural networks," IEEE Transactions on Neural Networks and Learning Systems, vol. 28, no. 1, pp. 206-217, 2017.

[11] Y. Guo, B. Ma, L. Chen, and R. Wu, "Adaptive sliding mode control for a class of Caputo type fractional-order interval systems with perturbation," IET Control Theory \& Applications, vol. 11, no. 1, pp. 57-65, 2017.

[12] X. Wu and H. Wang, "A new chaotic system with fractional order and its projective synchronization," Nonlinear Dynamics, vol. 61, no. 3, pp. 407-417, 2010.

[13] J. Hu, S. Chen, and L. Chen, "Adaptive control for antisynchronization of chua's chaotic system," Physics Letters A, vol. 339, no. 6, pp. 455-460, 2005.

[14] G. M. Mahmoud and E. E. Mahmoud, "Complete synchronization of chaotic complex nonlinear systems with uncertain parameters," Nonlinear Dynamics, vol. 62, no. 4, pp. 875-882, 2010.

[15] A. Khan and M. A. Bhat, "Multi-switching combination-combination synchronization of non-identical fractional-order chaotic systems," Mathematical Methods in the Applied Sciences, vol. 40, no. 15, pp. 5654-5667, 2017.

[16] J. Yu, C. Hu, H. Jiang, and X. Fan, "Projective synchronization for fractional neural networks," Neural Networks, vol. 49, pp. 87-95, 2014.

[17] P. Bagheri and M. Shahrokhi, "Neural network-based synchronization of uncertain chaotic systems with unknown states," Neural Computing and Applications, vol. 27, no. 4, pp. 945-952, 2016.

[18] S. Wang, Y. Huang, and S. Ren, "Synchronization and robust synchronization for fractional-order coupled neural networks," IEEE Access, vol. 5, pp. 12439-12448, 2017.

[19] H. Zhang, R. Ye, J. Cao, A. Ahmed, X. Li, and Y. Wan, "Lyapunov functional approach to stability analysis of riemann-liouville fractional neural networks with time-varying delays," Asian Journal of Control, vol. 20, no. 5, pp. 1938-1951, 2018.

[20] E. M. Shahverdiev, S. Sivaprakasam, and K. A. Shore, "Lag synchronization in time-delayed systems," Physics Letters A, vol. 292, no. 6, pp. 320-324, 2002.

[21] X. Li, C. Ding, and Q. Zhu, "Synchronization of stochastic perturbed chaotic neural networks with mixed delays," Journal of the Franklin Institute, vol. 347, no. 7, pp. 1266-1280, 2010.

[22] M. Zheng, L. Li, H. Peng, J. Xiao, Y. Yang, and H. Zhao, "Finite-time projective synchronization of memristor-based delay fractional-order neural networks," Nonlinear Dynamics, vol. 89, no. 4, pp. 2641-2655, 2017.

[23] K. Udhayakumar, R. Rakkiyappan, and G. Velmurugan, "Stability and synchronization of fractional-order complexvalued neural networks with time delay: LMI approach," The European Physical Journal Special Topics, vol. 226, no. 16-18, pp. 3639-3655, 2017.

[24] W. Wang, X. Jia, X. Luo, J. Kurths, and M. Yuan, "Fixed-time synchronization control of memristive MAM neural networks with mixed delays and application in chaotic secure communication," Chaos, Solitons \& Fractals, vol. 126, pp. 85-96, 2019.

[25] T. Hu, Z. He, X. Zhang, and S. Zhong, "Global synchronization of time-invariant uncertainty fractional-order neural networks with time delay," Neurocomputing, vol. 339, pp. 45-58, 2019.

[26] C. Wang, Q. Yang, Y. Zhuo, and R. Li, "Synchronization analysis of a fractional-order non-autonomous neural network with time delay," Physica A: Statistical Mechanics and Its Applications, vol. 549, Article ID 124176, 2020.

[27] X. You, Q. Song, and Z. Zhao, "Global Mittag-Leffler stability and synchronization of discrete-time fractional-order complex-valued neural networks with time delay," Neural Networks, vol. 122, pp. 382-394, 2020.

[28] Q. Song, S. Chen, Z. Zhao, Y. Liu, and F. E. Alsaadi, "Passive filter design for fractional-order quaternion-valued neural networks with neutral delays and external disturbance," Neural Networks, vol. 137, pp. 18-30, 2021.

[29] Y. Yuan, Q. Song, Y. Liu, and F. E. Alsaadi, "Synchronization of complex-valued neural networks with mixed two additive time-varying delays," Neurocomputing, vol. 332, pp. 149-158, 2019.

[30] L. Wang, Q. Song, Z. Zhao, Y. Liu, and F. E. Alsaadi, "Synchronization of two nonidentical complex-valued neural networks with leakage delay and time-varying delays," Neurocomputing, vol. 356, pp. 52-59, 2019.

[31] B. Hu, Q. Song, and Z. Zhao, "Robust state estimation for fractional-order complex-valued delayed neural networks with interval parameter uncertainties: LMI approach," $A p$ plied Mathematics and Computation, vol. 373, Article ID 125033, 2020.

[32] X. Yang, X. Wan, C. Zunshui, J. Cao, Y. Liu, and L. Rutkowski, "Synchronization of switched discrete-time neural networks via quantized output control with actuator fault," IEEE Transactions on Neural Networks and Learning Systems, pp. 1-11, 2020.

[33] X. Yang, X. Li, J. Lu, and Z. Cheng, "Synchronization of timedelayed complex networks with switching topology via hybrid actuator fault and impulsive effects control," IEEE Transactions on Cybernetics, vol. 50, no. 9, pp. 4043-4052, 2020.

[34] X. Yang, Y. Liu, J. Cao, and L. Rutkowski, "Synchronization of coupled time-delay neural networks with mode-dependent average dwell time switching," IEEE Transactions on Neural Networks and Learning Systems, vol. 31, no. 12, pp. 5483-5496, 2020.

[35] Q. Song, Y. Chen, Z. Zhao, Y. Liu, and F. E. Alsaadi, "Robust stability of fractional-order quaternion-valued neural networks with neutral delays and parameter uncertainties," Neurocomputing, vol. 420, pp. 70-81, 2021.

[36] S. Liang, R. Wu, and L. Chen, "Comparison principles and stability of nonlinear fractional-order cellular neural networks with multiple time delays," Neurocomputing, vol. 168, pp. 618-625, 2015.

[37] W. Zhang, R. Wu, R. Wu, J. Cao, A. Alsaedi, and T. Hayat, "Synchronization of a class of fractional-order neural networks with multiple time delays by comparison principles," Nonlinear Analysis: Modelling and Control, vol. 22, no. 5, pp. 636-645, 2017. 
[38] Y. Gu, Y. Yu, and H. Wang, "Projective synchronization for fractional-order memristor-based neural networks with time delays," Neural Computing and Applications, vol. 31, no. 10, pp. 6039-6054, 2019.

[39] X. Chen and Q. Song, "State estimation for quaternion-valued neural networks with multiple time delays," IEEE Transactions on Systems, Man, and Cybernetics: Systems, vol. 49, no. 11, pp. 2278-2287, 2019.

[40] I. Podlubny, Fractional Differential Equations, Academic Press, San Diego, CA, USA, 1999.

[41] V. Ladshmikantham and A. S. Vatsals, "Basic theory of fractional differential equations," Nonlinear Analysis: Theory, Methods \& Applications, vol. 60, pp. 2677-2682, 2008. 